\title{
Bond Additive Modeling 5. Mathematical Properties of the Variable Sum Exdeg Index
}

\author{
Damir Vukičević
}

\author{
Faculty of Natural Sciences and Mathematics, University of Split, Nikole Tesle 12, HR-21000 Split, Croatia
}

(E-mail:vukicevi@pmfst.hr)

RECEIVED JANUARY 13, 2010; REVISED JULY 22, 2010; ACCEPTED DECEMBER 2, 2010

\begin{abstract}
Recently, discrete and variable Adriatic indices have been introduced and it has been shown that the sum $\alpha$-exdeg index is good predictor (when variable parameter is equal to 0.37 ) of the octanol-water partition coefficient for octane isomers. Here, we study mathematical properties of this descriptor. Namely, we analyze extremal graphs of this descriptor in the following classes: the class of all connected graphs, the class of all trees, the class of all unicyclic graphs, the class of all chemical graphs, the class of all chemical trees, the class of all chemical unicyclic graphs, the class of all graphs with given maximal degree, the class of all graphs with given minimal degree, the class of all trees with given number of pendant vertices, and the class of all connected graphs with given number of pendant vertices. Also, many open problems about variable Adriatic indices are proposed. (doi: 10.5562/cca1667)
\end{abstract}

Keywords: molecular descriptor, extremal values, extremal graphs, chemical graphs, unicyclic graphs, trees

\section{INTRODUCTION}

Recently, discrete and variable Adriatic indices have been introduced ${ }^{1}$ and studied. Predictive and mathematical properties of discrete Adriatic indices have been analyzed in papers. ${ }^{1,2}$ Predictive properties of variable Adriatic indices have been studied in Ref. 3. It has been found that three variable Adriatic indices have especially good predictive properties, namely:

1) the inverse sum -1.95-deg index is well correlated with standard enthalpy of formation of octane isomers $\left(R^{2}=0.75\right)$

2) the inverse sum 0.43-lodeg index is well correlated with total surface area of octane isomers $\left(R^{2}=0.92\right)$

3) the sum 0.37-exdeg index is well correlated with the octanol-water partition coefficient $\left(R^{2}=0.99\right)$.

In this paper, we restrict our attention to the variable sum exdeg index. The variable sum exdeg index is defined by:

$$
S E I_{a}(G)=\sum_{u v \in E(G)}\left(a^{d_{u}}+a^{d_{v}}\right), a \in(0,1) \cup(1,+\infty) .
$$

where $E(G)$ is the set of edges of $G$. Note that this index can be rewritten as:

$$
\begin{aligned}
\operatorname{SEI}_{a}(G)= & \sum_{u v \in E(G)}\left(a^{d_{u}}+a^{d_{v}}\right)= \\
& \sum_{u \in V(G)} \sum_{v \in V(G): u v \in E(G)} a^{d_{u}}=\sum_{u \in V(G)} d_{u} \cdot a^{d_{u}},
\end{aligned}
$$

where $V(G)$ is the set of vertices of $G$. Hence, this index can be considered as a sum of vertex contributions such that the contribution of each vertex depends solely on its degree. One immediately see a parallel to the well known the first Zagreb index ${ }^{4}$ defined by:

$$
M_{1}(G)=\sum_{u \in V(G)} d_{u}^{2} .
$$

The mathematical and predictive properties of Zagreb index have been extensively studied (see Refs. 4-6 and references within and, for recent mathematical studies, Refs. 7-12).

In this paper, we analyze graphs with extremal values of the $S E I_{a}$ index in the following classes: the class of all connected graphs, the class of all trees, the class of all unicyclic graphs, the class of all chemical graphs, the class of all chemical trees, the class of all 
chemical unicyclic graphs, the class of all graphs with given maximal degree, the class of all graphs with given minimal degree, the class of all trees with given number of pendant vertices, and the class of all connected graphs with given number of pendant vertices.

These results can be used for the detection of chemical compounds that may have desirable properties. Namely, if one finds some property well-correlated with this descriptor for some value of $\alpha$, then extremal graphs should correspond to molecules with minimal or maximal value of that property. Since one such property has already been found, ${ }^{3}$ this may encourage the further study of this index.

\section{MATHEMATICAL PROPERTIES OF SUM $a$ - EXDEG INDEX FOR $a>1$}

In this paper, we consider only simple connected graphs, so from now on by graph we imply simple connected graph. The number of vertices of $G$ will be denoted by $n(G)$ and number of edges by $m(G)$. By $n_{i}(G)$ we denote the number of vertices of degree $i$. Let $f_{a}:[1,+\infty) \rightarrow \mathbb{R}$ be the function defined by $f_{a}(x)=x \cdot a^{x}$, where $a>0$. It can be easily seen that:

Lemma 1. Function $f_{a}$ is an increasing function for each $a>1$.

Proof: Inequality $f_{a}{ }^{\prime}(x)=a^{x}+a^{x} \cdot x \cdot \ln a>0 \quad$ implies the Lemma.

Lemma 2. Function $f_{a}$ is a convex function for each $a>1$.

Proof:Inequality $f_{a}{ }^{\prime \prime}(x)=2 a^{x} \ln a+a^{x} \cdot x \cdot \ln ^{2} a>0$ implies the Lemma.

From the Lemma 1, it directly follows that:

Proposition 3. $S E I_{a}(G) \leq n \cdot(n-1) \cdot a^{n-1}$ for every graph $G$ with $n$ vertices and for each $a>1$. Equality holds if and only if $G$ is a complete graph.

Proposition 4. $S E I_{a}(G) \leq n \cdot \Delta \cdot a^{\Delta}$ for every graph $G$ with $n$ vertices and maximal degree $\Delta$; and for each $a>1$. Equality holds if and only if $G$ is a $\Delta$-regular graph.

Proposition 5. $S E I_{a}(G) \leq n \cdot 4 \cdot a^{4}$ for every chemical graph $G$ with $n$ vertices and for each $a>1$. Equality holds if and only if $G$ is a 4-regular graph.

Proposition 6. $n \cdot \delta \cdot a^{\delta} \leq S E I_{a}(G) \leq \delta \cdot a^{\delta}+\delta \cdot(n-1) \cdot a^{n-1}$ $+(n-1-\delta) \cdot(n-2) \cdot a^{n-2}$ for every graph $G$ with $n$ vertices and minimal degree $\delta$; and for each $a>1$. Equality holds for the lower bound if and only if $G$ is a $\delta$-regular graph. Equality holds for the upper bound if and only if $G$ has a vertex $u$ of degree $\delta$ and $G-u$ is a complete graph on $n-1$ vertices.

Let us prove:
Lemma 7. Let $x_{1}, \ldots, x_{n} \in\{1, \ldots, n-1\}$ be numbers such that $x_{1}+\ldots+x_{n}=2 n-2$ and let $a>1$. Then

$2 \cdot a+(n-2) \cdot 2 \cdot a^{2} \leq \sum_{i=1}^{n} f\left(x_{i}\right) \leq(n-1) \cdot a+(n-1) \cdot a^{n-1}$.

The equality holds for the lower bound if and only if the following multisets are equal $\left\{x_{1}, \ldots x_{n}\right\}=\left\{1_{2}, 2_{n-2}\right\}$ and the equality holds for the upper bound if and only if $\left\{x_{1}, \ldots, x_{n}\right\}=\left\{(n-1)_{1}, 1_{n-1}\right\}$.

Proof: First, let us prove the lower bound. It can be easily seen that equality holds for $\left\{x_{1}, \ldots x_{n}\right\}=\left\{1_{2}, 2_{n-2}\right\}$.

Let $\left\{x_{1}, \ldots, x_{n}\right\}$ be the $n$-tuple with the smallest value

of $\sum_{i=1}^{n} f\left(x_{i}\right)$. Suppose to the contrary that $\left\{x_{1}, \ldots x_{n}\right\} \neq\left\{1_{2}, 2_{n-2}\right\}$. Without loss of generality, we may assume that $x_{1} \leq x_{2} \leq \ldots \leq x_{n}$. It can be easily seen that $x_{1}=1$ and that $x_{n} \geq 3$, but then

$$
\begin{aligned}
& f\left(x_{1}+1\right)+f\left(x_{2}\right)+f\left(x_{3}\right)+\ldots+f\left(x_{n-1}\right)+f\left(x_{n}-1\right)= \\
& =\sum_{i=1}^{n} f\left(x_{i}\right)+\left[f\left(x_{1}+1\right)+f\left(x_{n}-1\right)\right]-\left[f\left(x_{1}\right)+f\left(x_{n}\right)\right]< \\
& <\sum_{i=1}^{n} f\left(x_{i}\right),
\end{aligned}
$$

because $f$ is convex. This is in contradiction with the choice of $\left\{x_{1}, \ldots, x_{n}\right\}$.

Now, let us prove the upper bound. It can be easily seen that the equality holds for $\left\{x_{1}, \ldots, x_{n}\right\}=$ $\left\{(n-1), 1_{n-1}\right\}$. Let $\left\{x_{1}, \ldots, x_{n}\right\}$ be the $n$-tuple with the highest value of $\sum_{i=1}^{n} f\left(x_{i}\right)$. Suppose to the contrary that $\left\{x_{1}, \ldots, x_{n}\right\} \neq\left\{(n-1)_{1}, 1_{n-1}\right\}$. Without loss of generality, we may assume that $x_{1} \leq x_{2} \leq \ldots \leq x_{n}$. It can be easily seen that $x_{n} \leq n-2$ and that $x_{n-1} \geq 2$, but then

$$
\begin{aligned}
& f\left(x_{1}\right)+f\left(x_{2}\right)+\ldots+f\left(x_{n-1}\right)+f\left(x_{n-1}-1\right)+f\left(x_{n}+1\right)= \\
& =\sum_{i=1}^{n} f\left(x_{i}\right)+\left[f\left(x_{n-1}-1\right)+f\left(x_{n}+1\right)\right]-\left[f\left(x_{n-1}\right)+f\left(x_{n}\right)\right]> \\
& >\sum_{i=1}^{n} f\left(x_{i}\right),
\end{aligned}
$$

because $f$ is convex. This is in contradiction with the choice of $\left\{x_{1}, \ldots, x_{n}\right\}$.

From here, it directly follows that: 
Proposition 8. $2 \cdot a+(n-2) \cdot 2 \cdot a^{2} \leq S E I_{a}(G) \leq(n-1) \cdot a+$ $(n-1) \cdot a+(n-1) \cdot a^{n-1}$ for each tree $G$ with $n$ vertices and for each $a>1$. The equality for the lower bound holds if and only if $G$ is a path. The equality holds for the upper bound holds if and only if $G$ is a star $S_{n}$.

Proof: Note that $1 \leq d_{u} \leq n-1$ for each vertex $u \in V(G)$ and that $\sum_{u \in V(G)} d_{u}=2 n-2$.

Let us recall that every graph $G$ contains a spanning tree $T$, i.e. subgraph $T$ which is a tree such that $V(G)=V(T)$. Let us prove:

Lemma 9. Let $G$ be graph and $T$ its spanning tree, then $S E I_{a}(G) \geq S E I_{a}(T)$ for each $a>1$.

Proof: Since the degree of every vertex in $T$ is not larger then in $G$, the Lemma follows.

From Proposition 8 and Lemma 9, it follows:

Corollary 9. $S E I_{a}(G) \geq 2 \cdot a+(n-2) \cdot 2 \cdot a^{2}$ for each graph $G$ with $n$ vertices and for each $a>1$. The equality for the lower bound holds if and only if $G$ is a path.

Corollary 10. $\operatorname{SEI}_{a}(G) \geq 2 \cdot a+(n-2) \cdot 2 \cdot a^{2}$ for each chemical graph $G$ with $n$ vertices and for each $a>1$. The equality for the lower bound holds if and only if $G$ is a path.

Denote by $A_{n}$ set of all multisets $\left\{x_{1}, \ldots, x_{n}\right\}$ such that:

1) $x_{1}, \ldots, x_{n} \in\{1, \ldots, n-1\}$;

2) $x_{1}+\ldots+x_{n}=2 n$

3) at least three numbers $x_{i}$ are greater then 1 .

Let us prove:

Lemma 11. Let $\left\{x_{1}, \ldots, x_{n}\right\} \in A_{n}$. Then

$$
2 n \cdot a^{2} \leq \sum_{i=1}^{n} f\left(x_{i}\right) \leq(n-1) \cdot a^{n-1}+4 \cdot a^{2}+(n-3) \cdot a .
$$

The equality holds for the lower bound if and only if $\left\{x_{1}, \ldots x_{n}\right\}=\left\{2_{n}\right\}$ and the equality for the upper bound holds if and only if $\left\{x_{1}, \ldots, x_{n}\right\}=\left\{1_{n-3}, 2_{2},(n-1)_{1}\right\}$.

Proof: First, let us prove the lower bound. It can be easily seen that equality holds for $\left\{x_{1}, \ldots x_{n}\right\}=\left\{2_{n}\right\}$. Let $\left\{x_{1}, \ldots, x_{n}\right\} \in A_{n}$ be the $n$-tuple with smallest value of $\sum_{i=1}^{n} f\left(x_{i}\right)$. Suppose to the contrary that $\left\{x_{1}, \ldots x_{n}\right\} \neq$ $\left\{2_{n}\right\}$. Without loss of generality, we may assume that $x_{1} \leq x_{2} \leq \ldots \leq x_{n}$. It can be easily seen that $x_{1}=1$ and that $x_{n} \geq 3$, but then:

$$
\begin{aligned}
& f\left(x_{1}+1\right)+f\left(x_{2}\right)+f\left(x_{3}\right)+\ldots+f\left(x_{n-1}\right)+f\left(x_{n}-1\right)= \\
& =\sum_{i=1}^{n} f\left(x_{i}\right)+\left[f\left(x_{1}+1\right)+f\left(x_{n}-1\right)\right]-\left[f\left(x_{1}\right)+f\left(x_{n}\right)\right]<
\end{aligned}
$$

$<\sum_{i=1}^{n} f\left(x_{i}\right)$

because function $f$ is convex. From the choice of $\left\{x_{1}, \ldots, x_{n}\right\}$, it follows that $\left\{x_{1}+1, x_{2}, x_{3}, \ldots, x_{n-1}, x_{n}-1\right\} \in$ $A_{n}$, i.e. that it has at least 3 elements larger then 1 , but this is not possible.

Now, let us prove the upper bound. It can be easily seen that equality holds for $\left\{x_{1}, \ldots, x_{n}\right\}=$ $\left\{1_{n-3}, 2_{2},(n-1)_{1}\right\}$. Let $\left\{x_{1}, \ldots, x_{n}\right\} \in A_{n}$ be the $n$-tuple with highest value of $\sum_{i=1}^{n} f\left(x_{i}\right)$. Suppose to the contrary that $\left\{x_{1}, \ldots, x_{n}\right\} \neq\left\{1_{n-3}, 2_{2},(n-1)_{1}\right\}$. Without loss of generality, we may assume that $x_{1} \leq x_{2} \leq \ldots \leq x_{n}$. It can be easily seen that $x_{n} \leq n-2$ and that $x_{n-1} \geq 2$, but then

$f\left(x_{1}\right)+f\left(x_{2}\right)+\ldots+f\left(x_{n-1}\right)+f\left(x_{n-1}-1\right)+f\left(x_{n}+1\right)=$

$=\sum_{i=1}^{n} f\left(x_{i}\right)+\left[f\left(x_{n-1}-1\right)+f\left(x_{n}+1\right)\right]-\left[f\left(x_{n-1}\right)+f\left(x_{n}\right)\right]>$

$>\sum_{i=1}^{n} f\left(x_{i}\right)$

because $f$ is convex. From the choice of $\left\{x_{1}, \ldots, x_{n}\right\}$, it follows that

$$
\left\{x_{1}, x_{2}, x_{3}, \ldots, x_{n-1}-1, x_{n}+1\right\} \notin A_{n},
$$

i.e. that it has less then 3 elements larger then 1 . This is possible only if $x_{1}=x_{2}=\ldots=x_{n-3}=1$ and $x_{n-2}=$ $x_{n-1}=2$, but then simple calculation gives $x_{n}=n-1$, i.e. $\left\{x_{1}, \ldots, x_{n}\right\}=\left\{1_{n-3}, 2_{2},(n-1)_{1}\right\}$, which is a contradiction.

Let $S_{n}^{+}$be the graph obtained from the star $S_{n}$ by adding an edge connecting two leaves as presented in the Figure 1.

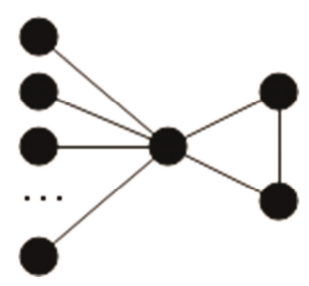

Figure 1. Graph $A_{n}$. 
From Lemma 11, it directly follows that:

Proposition 12. $2 n \cdot a^{2} \leq S E I_{a}(G) \leq(n-1) \cdot a^{n-1}+4 \cdot a^{2}+$ $(n-3) \cdot a$ for every unicyclic graph $G$ with $n$ vertices and for each $a>1$. E quality for the lower bound holds if and only if $G$ is a cycle $C_{n}$. Equality for the upper bound holds if and only if $G=S_{n}^{+}$.

Proof: Denote $V=\left\{v_{1}, \ldots v_{n}\right\}$ and note that:

1) $d_{v_{1}}, \ldots, d_{v_{n}} \in\{1, \ldots, n-1\}$;

2) $d_{v_{1}}+\ldots+d_{v_{n}}=2 n$

3) at least three numbers $d_{v_{i}}$ are greater then 1 .

Let $S_{n, k}$ be a class of graphs with $n$ vertices obtained from $S_{k+1}$ by replacing some edges by paths. Similarly as above, it can be proved that:

Lemma 13. Let $\Delta \in \mathbb{N}$ and let $x_{1}, \ldots, x_{n-1} \in\{1, \ldots, n-1\}$ be numbers such that $x_{1}+\ldots+x_{n-1}=2 n-2-\Delta$ and let $a>1$. Then

$$
\sum_{i=1}^{n-1} f\left(x_{i}\right) \geq \Delta \cdot a+2 \cdot(n-\Delta-1) \cdot a^{2} .
$$

Equality holds if and only if $\left\{x_{1}, \ldots, x_{n}\right\}=\left\{1_{\Delta}, 2_{n-\Delta-1}\right\} \cdot \square$ From this Lemma, it follows:

Proposition 14. $S E I_{a}(G) \geq \Delta \cdot a+2 \cdot(n-\Delta-1) \cdot a^{2}+$ $\Delta \cdot a^{\Delta}$ for every graph $G$ with $n$ vertices and maximal degree $\Delta$, and for each $a>1$. Equality holds if and only if $G \in S_{n, k}$

Proof: From Lemma 9, it follows that it is sufficient to prove the claim for trees. Hence, let us assume that $G$ is tree. Denote $V(G)=\left\{v_{1}, \ldots, v_{n}\right\}$ where $d_{v_{n}}=\Delta$. It is sufficient to note that $\left\{d_{v_{1}}, \ldots, d_{v_{n-1}}\right\}$ satisfy the condition of the previous Lemma. Moreover, the equality holds if and only if $\left\{d_{v_{1}}, \ldots, d_{v_{n-1}}\right\}=\left\{1_{\Delta}, 2_{n-\Delta-1}\right\}$, but this is possible if and only if $G \in S_{n, k}$.

Using similar techniques as above, it can be proved that:

Proposition 15. It holds that

$$
\begin{aligned}
& k \cdot a+(n-2 k+2) \cdot 2 \cdot a^{2}+(k-2) \cdot 3 \cdot a^{3} \leq \operatorname{SEI}_{a}(G) \leq \\
& \leq k \cdot a+2 \cdot(n-k-1) \cdot a^{2}+k \cdot a^{k}
\end{aligned}
$$

for every tree $T$ with $n$ vertices and $k$ pendant vertices, and for each $a>1$. The equality for the lower bound holds if and only if $G$ is a graph with $k$ vertices of degree $1, n-2 k+2$ vertices of degree 2 and $k-2$ vertices of degree 3 . The equality for the upper bound holds if and only if $G \in S_{n, k}$. $\square$

Proposition 16. It holds

$$
\begin{aligned}
& k \cdot a+(n-2 k+2) \cdot 2 \cdot a^{2}+(k-2) \cdot 3 \cdot a^{3} \leq \operatorname{SEI}_{a}(G) \leq \\
& \leq k \cdot a+(n-1) \cdot a^{n-1}+(n-k-1)^{2} \cdot a^{n-k-1}
\end{aligned}
$$

for every graph $G$ with $n$ vertices and $k$ pendant vertices, and for each $a>1$. The equality for the lower bound holds if and only if $G$ is a graph with $k$ vertices of degree $1, n-2 k+2$ vertices of degree 2 and $k-2$ vertices of degree 3 . The equality holds for the upper bound if and only if all pendant vertices are adjacent to the same vertex, and the subgraph obtained by elimination of pendant vertices is a complete graph $\square$

Proposition 17.2 $a+(n-2) \cdot 2 \cdot a^{2} \leq S E I_{a}(G) \leq$ $\frac{2 n+2}{3} \cdot a+\frac{n-2}{3} \cdot 4 \cdot a^{4}$ for each chemical tree $G$ with $n$ vertices, and for each $a>1$. The equality holds for the lower bound holds if and only if $G$ is a path. The equality holds for the upper bound if and only if $G$ has only vertices of degree 1 and 4 .

Proposition 18. $2 n \cdot a^{2} \leq S E I_{a}(G) \leq \frac{2 n}{3} \cdot a+\frac{n}{3} \cdot 4 \cdot a^{4}$

for each chemical unicyclic graph $G$ with $n$ vertices and for each $a>1$. The equality holds for the lower bound holds if and only if $G$ is a cycle. The equality holds for the upper bound if and only if $G$ has only vertices of degree 1 and 4.

\section{MATHEMATICAL PROPERTIES OF SUM $a$ - EXDEG INDEX FOR $a<1$}

Here, we restrict our attention to chemical graphs. First, let us analyze chemical trees.

Theorem 19. Let $a \in(0,1)$ and $G$ be a chemical tree with $n$ vertices. It holds:

$S E I_{a}(G) \geq\left\{\begin{aligned}(n-2) \cdot 2 a^{2}+2 a, & \text { if } 0<a \leq \frac{1}{3} ; \\ (n-2) \cdot \frac{3 a^{3}+a}{2}+2 a, & \text { if } \\ \frac{1}{3} \leq a_{2} \leq \frac{3}{4} \cdot \cos \left(\frac{\pi+\operatorname{Arccos}\left(\frac{5}{27}\right)}{3}\right)+\frac{3}{8} & \text { if } \\ (n-2) \cdot \frac{4 a^{4}+2 a}{3}+2 a, & \frac{3}{4} \cdot \cos \left(\frac{\pi+\operatorname{Arccos}\left(\frac{5}{27}\right)}{3}\right)+\frac{3}{8}<a<1 ;\end{aligned}\right.$ 
$\operatorname{SEI}_{a}(G) \leq\left\{\begin{array}{cc}(n-2) \cdot \frac{4 a^{4}+2 a}{3}+2 a, & \text { if } 0<a \leq-\sqrt{2} \cdot \cos \frac{7 \pi}{12} \\ (n-2) \cdot 2 a^{2}+2 a, & \text { if }-\sqrt{2} \cdot \cos \frac{7 \pi}{12}<a<1 .\end{array}\right.$

Moreover, the equality in the lower bounds holds if and only if one of the following holds:

a) $\quad a \in\left(0, \frac{1}{3}\right)$ and $G$ is a path $P_{n}$;

b) $\quad a=\frac{1}{3}$ and $G$ only has vertices of degrees 1,2 and 3;

c) $a \in\left(\frac{1}{3}, \frac{3}{4} \cdot \cos \left(\frac{\pi+\operatorname{Arccos}\left(\frac{5}{27}\right)}{3}\right)+\frac{3}{8}\right)$ and $G$ only has vertices of degrees 1 and 3 ;

d) $a=\frac{3}{4} \cdot \cos \left(\frac{\pi+\operatorname{Arccos}\left(\frac{5}{27}\right)}{3}\right)+\frac{3}{8}$ and $G$ only has vertices of degrees 1,3 and 4;

e) $a \in\left(\frac{3}{4} \cdot \cos \left(\frac{\pi+\operatorname{Arccos}\left(\frac{5}{27}\right)}{3}\right)+\frac{3}{8}, 1\right)$ and $G$ only has vertices of degrees 1 and 4 ;

the equality in the upper bounds holds if and only if one of the following holds: a) $\quad a \in\left(0,-\sqrt{2} \cdot \cos \frac{7 \pi}{12}\right)$ and $G$ only has vertices of degrees 1 and 4

b) $a=-\sqrt{2} \cdot \cos \frac{7 \pi}{12}$ and $G$ only has vertices of degrees 1, 2 and 4;

c) $\quad a \in\left(-\sqrt{2} \cdot \cos \frac{7 \pi}{12}, 1\right)$ and $G$ is a path $P_{n}$.

Proof: Note that $n_{1}(G)=n_{3}(G)+2 n_{4}(G)+2$. Hence, Moreover, the equality for the upper bound holds if and only if:

1) $n_{2}(G)=0$ or

$$
2 a^{2}=\max \left\{2 a^{2}, \frac{3 a^{3}+a}{2}, \frac{4 a^{4}+2 a}{3}\right\} ;
$$

2) $n_{3}(G)=0$ or

$$
\frac{3 a^{3}+a}{2}=\max \left\{2 a^{2}, \frac{3 a^{3}+a}{2}, \frac{4 a^{4}+2 a}{3}\right\} ;
$$

3) $n_{4}(G)=0$ or

$$
\frac{4 a^{4}+2 a}{3}=\max \left\{2 a^{2}, \frac{3 a^{3}+a}{2}, \frac{4 a^{4}+2 a}{3}\right\} ;
$$

and the inequality for the lower bound holds if and only if:

I) $n_{2}(G)=0$ or $2 a^{2}=\min \left\{2 a^{2}, \frac{3 a^{3}+a}{2}, \frac{4 a^{4}+2 a}{3}\right\}$;

II) $n_{3}(G)=0$ or

$$
\begin{aligned}
& \operatorname{SEI}_{a}(G)=n_{1}(G) \cdot a+n_{2}(G) \cdot 2 a^{2}+n_{3}(G) \cdot 3 a^{3}+n_{4}(G) \cdot 4 a^{4} \\
& \operatorname{SEI}_{a}(G)-2 a=\left(n_{1}(G)-2\right) \cdot a+n_{2}(G) \cdot 2 a^{2}+n_{3}(G) \cdot 3 a^{3}+n_{4}(G) \cdot 4 a^{4} \\
& \frac{\operatorname{SEI}_{a}(G)-2 a}{n-2}=\frac{\left[n_{3}(G)+2 n_{4}(G)+2-2\right] \cdot a+n_{2}(G) \cdot 2 a^{2}+n_{3}(G) \cdot 3 a^{3}+n_{4}(G) \cdot 4 a^{4}}{\left[n_{3}(G)+2 n_{4}(G)+2\right]+n_{2}(G)+n_{3}(G)+n_{4}(G)-2} \\
& \frac{\operatorname{SEI}_{a}(G)-2 a}{n-2}=\frac{n_{2}(G) \cdot 2 a^{2}+n_{3}(G) \cdot\left(3 a^{3}+a\right)+n_{4}(G) \cdot\left(4 a^{4}+2 a\right)}{n_{2}(G)+2 n_{3}(G)+3 n_{4}(G)} .
\end{aligned}
$$

Note that

$$
\begin{aligned}
& \min \left\{\frac{2 a^{2}}{1}, \frac{3 a^{3}+a}{2}, \frac{4 a^{4}+2 a}{3}\right\} \leq \frac{n_{2}(G) \cdot 2 a^{2}+n_{3}(G) \cdot\left(3 a^{3}+a\right)+n_{4}(G) \cdot\left(4 a^{4}+2 a\right)}{n_{2}(G)+2 n_{3}(G)+3 n_{4}(G)} \\
& \frac{n_{2}(G) \cdot 2 a^{2}+n_{3}(G) \cdot\left(3 a^{3}+a\right)+n_{4}(G) \cdot\left(4 a^{4}+2 a\right)}{n_{2}(G)+2 n_{3}(G)+3 n_{4}(G)} \leq \max \left\{2 a^{2}, \frac{3 a^{3}+a}{2}, \frac{4 a^{4}+2 a}{3}\right\} .
\end{aligned}
$$




$$
\frac{3 a^{3}+a}{2}=\min \left\{2 a^{2}, \frac{3 a^{3}+a}{2}, \frac{4 a^{4}+2 a}{3}\right\} ;
$$

III) $n_{4}(G)=0$ or

$$
\frac{4 a^{4}+2 a}{3}=\min \left\{2 a^{2}, \frac{3 a^{3}+a}{2}, \frac{4 a^{4}+2 a}{3}\right\} .
$$

Therefore:

$$
\begin{aligned}
& \min \left\{2 a^{2}, \frac{3 a^{3}+a}{2}, \frac{4 a^{4}+2 a}{3}\right\} \leq \frac{S E I_{a}(G)-2 a}{n-2} \leq \\
& \leq \max \left\{2 a^{2}, \frac{3 a^{3}+a}{2}, \frac{4 a^{4}+2 a}{3}\right\},
\end{aligned}
$$

which implies

$$
\begin{aligned}
& \operatorname{SEI}_{a}(G) \leq(n-2) \cdot \max \left\{2 a^{2}, \frac{3 a^{3}+a}{2}, \frac{4 a^{4}+2 a}{3}\right\}+2 a ; \\
& S E I_{a}(G) \geq(n-2) \cdot \min \left\{2 a^{2}, \frac{3 a^{3}+a}{2}, \frac{4 a^{4}+2 a}{3}\right\}+2 a .
\end{aligned}
$$

Moreover, the equality for the upper bound holds if and only if conditions 1)-3) hold and the equality for the lower bound holds if and only if conditions I)-III) hold. In order to prove the theorem, we need to find $\min \left\{2 a^{2}, \frac{3 a^{3}+a}{2}, \frac{4 a^{4}+2 a}{3}\right\}$ and $\max \left\{2 a^{2}, \frac{3 a^{3}+a}{2}, \frac{4 a^{4}+2 a}{3}\right\}$.

Solving equation $2 a^{2}=\frac{3 a^{3}+a}{2}$ for $a \in(0,1)$, we get $a_{1}=\frac{1}{3} \approx 0.333$. Solving $2 a^{2}=\frac{4 a^{4}+2 a}{3}$ for $a \in(0,1)$, we get $a_{2}=-\sqrt{2} \cdot \cos \frac{7 \pi}{12} \approx 0.366$. Finally, solving equation $\frac{3 a^{3}+a}{2}=\frac{4 a^{4}+2 a}{3}$ for $a \in(0,1)$, we get

$$
a_{3}=\frac{3}{4} \cdot \cos \left(\frac{\pi+\operatorname{Arccos}\left(\frac{5}{27}\right)}{3}\right)+\frac{3}{8} \approx 0.4215
$$

Now, we write the table of (approximate) values of the function at zero points and some arbitrary points (one smaller than all of them, one between each two successive zero points and one larger than all of them). (see Table 1)

From this table (Table 1), we can easily determine $\min \left\{2 a^{2}, \frac{3 a^{3}+a}{2}, \frac{4 a^{4}+2 a}{3}\right\}$ and $\max \left\{2 a^{2}, \frac{3 a^{3}+a}{2}, \frac{4 a^{4}+2 a}{3}\right\}$

Completely analogously, it can be proved that:

Theorem 20. Let $a \in(0,1)$ and $G$ be a unicyclic chemical graph with $n$ vertices. It holds:

$$
S E I_{a}(G) \geq\left\{\begin{array}{l}
n \cdot 2 a^{2}, \quad \text { if } 0<a \leq \frac{1}{3} \\
n \cdot \frac{3 a^{3}+a}{2}, \quad \text { if } \\
\frac{1}{3} \leq a_{2} \leq \frac{3}{4} \cdot \cos \left(\frac{\pi+\operatorname{Arccos}\left(\frac{5}{27}\right)}{3 \cdot \frac{4 a^{4}+2 a}{3},} \quad \text { if }\right)+\frac{3}{8} \\
\frac{3}{4} \cdot \cos \left(\frac{\pi+\operatorname{Arc~} \cos \left(\frac{5}{27}\right)}{3}\right)+\frac{3}{8}<a<1
\end{array}\right.
$$

Table 1. Function values in significant points

\begin{tabular}{ccccccccc} 
& 0.1 & $a_{1}$ & 0.35 & $a_{2}$ & 0.4 & $a_{3}$ & 0.6 \\
$2 a^{2}$ & 0.02 & 0.222 & 0.245 & 0.268 & 0.32 & 0.355 & 0.72 \\
$\frac{3 a^{3}+a}{2}$ & 0.052 & 0.222 & 0.239 & 0.257 & 0.296 & 0.323 & 0.624 \\
$\frac{4 a^{4}+2 a}{3}$ & 0.067 & 0.239 & 0.253 & 0.268 & 0.301 & 0.323 & 0.573 \\
\hline
\end{tabular}


$S E I_{a}(G) \leq\left\{\begin{array}{cc}n \cdot \frac{4 a^{4}+2 a}{3}, & \text { if } 0<a \leq-\sqrt{2} \cdot \cos \frac{7 \pi}{12} \\ n \cdot 2 a^{2}, & \text { if }-\sqrt{2} \cdot \cos \frac{7 \pi}{12}<a<1 .\end{array}\right.$

Moreover, the equality in the lower bounds holds if and only if one of the following holds:

a) $\quad a \in\left(0, \frac{1}{3}\right)$ and $G$ is a cycle $C_{n}$;

b) $\quad a=\frac{1}{3}$ and $G$ only has vertices of degrees 1,2 and 3;

c) $a \in\left(\frac{1}{3}, \frac{3}{4} \cdot \cos \left(\frac{\pi+\operatorname{Arccos}\left(\frac{5}{27}\right)}{3}\right)+\frac{3}{8}\right)$ and $G$ only has vertices of degrees 1 and 3 ;

d) $a=\frac{3}{4} \cdot \cos \left(\frac{\pi+\operatorname{Arccos}\left(\frac{5}{27}\right)}{3}\right)+\frac{3}{8}$ and $G$ only has vertices of degrees 1,3 and 4 ;

e) $a \in\left(\frac{3}{4} \cdot \cos \left(\frac{\pi+\operatorname{Arccos}\left(\frac{5}{27}\right)}{3}\right)+\frac{3}{8}, 1\right)$ and $G$ only has vertices of degrees 1 and 4;

the equality in the upper bounds holds if and only if one of the following holds:

a) $a \in\left(0,-\sqrt{2} \cdot \cos \frac{7 \pi}{12}\right)$ and $G$ only has vertices of degrees 1 and 4 ;

b) $a=-\sqrt{2} \cdot \cos \frac{7 \pi}{12}$ and $G$ only has vertices of degrees 1,2 and 4

c) $a \in\left(-\sqrt{2} \cdot \cos \frac{7 \pi}{12}, 1\right)$ and $G$ is a cycle $C_{n}$.

Theorem 21. Let $a \in(0,1)$ and $G$ be a chemical graph with $n$ vertices. It holds:

1) $\operatorname{SEI}_{a}(G) \leq 4 n \cdot a^{4}$ for $a \in\left(\frac{3}{4}, 1\right)$ with equality if and only if $G$ is a 4-regular graph;

2) $S E I_{a}(G) \leq 4 n \cdot a^{4}$ for $a=\frac{3}{4}$ with equality if and only if all vertices in $G$ have degree 3 or 4;
3) $S E I_{a}(G) \leq 3 n \cdot a^{3}$ for $a \in\left(\frac{2}{3}, \frac{3}{4}\right)$ with equality if and only if $G$ is a 3-regular graph;

4) $S E I_{a}(G) \leq 3 n \cdot a^{3}$ for $a=\frac{2}{3}$ with equality if and only if all vertices in $G$ have degree 2 or 3;

5) $\operatorname{SEI}_{a}(G) \leq 2 n \cdot a^{2}$ for $a \in\left(\frac{1}{2}, \frac{2}{3}\right)$ with equality if and only if $G$ is a 2-regular graph;

6) $\operatorname{SEI}_{a}(G) \leq 2 n \cdot a^{2}$ for $a=\frac{1}{2}$ with equality if $G$ is $P_{n}$ or $C_{n}$;

7) $\operatorname{SEI}_{a}(G) \leq(n-2) \cdot 2 a^{2}+2 a$ for

$a \in\left(-\sqrt{2} \cdot \cos \frac{7 \pi}{12}, \frac{1}{2}\right)$ with equality if and only if $G$ is a path $P_{n}$;

8) $\operatorname{SEI}_{a}(G) \leq(n-2) \cdot 2 a^{2}+2 a$ for $a=-\sqrt{2} \cdot \cos \frac{7 \pi}{12}$ with equality if and only if $G$ is a tree that has only vertices of degrees 1,2 and 4 ;

9) $\operatorname{SEI}_{a}(G) \leq(n-2) \cdot \frac{4 a^{4}+2 a}{3}+2 a$ for $a \in\left(0,-\sqrt{2} \cdot \cos \frac{7 \pi}{12}\right)$ with equality if and only if $G$ is a tree that has only vertices of degrees 1 and 4 .

Proof: In order to prove 1)-6), it is sufficient to note that:

1') $4 a^{4}>\max \left\{3 a^{3}, 2 a^{2}, a\right\}$ for $a \in\left(\frac{3}{4}, 1\right)$;

2) $4 a^{4}=3 a^{3}>\max \left\{2 a^{2}, a\right\}$ for $a=\frac{3}{4}$;

3') $3 a^{3}>\max \left\{4 a^{4}, 2 a^{2}, a\right\}$ for $a \in\left(\frac{2}{3}, \frac{3}{4}\right)$;

4) $3 a^{3}=2 a^{2}>\max \left\{4 a^{4}, a\right\}$ for $a=\frac{2}{3}$;

5') $2 a^{2}>\max \left\{a, 3 a^{3}, 4 a^{4}\right\}$ for $a \in\left(\frac{1}{2}, \frac{2}{3}\right)$;

6) $2 a^{2}=a>\max \left\{3 a^{4}, 4 a^{4}\right\}$ for $a=\frac{1}{2}$.

Now, let us assume that $a<\frac{1}{2}$. Let $T$ be a spanning tree of $G$. Since, $a>2 a^{2}>3 a^{3}>4 a^{4}$ and the degrees of all vertices in $T$ are not greater than in $G$ (with all equalities if and only if $G=T$ ), it follows that the maximum is achieved if and only if $G=T$. Claims 7)-9) follow from the Theorem 18 .

Theorem 22. Let $a \in(0,1)$ and $G$ be a chemical graph 
with $n$ vertices. It holds:

1) $S E I_{a}(G) \geq 4 n \cdot a^{4}$ for $a \in\left(0, \sqrt[3]{\frac{1}{4}}\right)$ with equality if and only if $G$ is a 4-regular graph;

2) $S E I_{a}(G) \geq 4 n \cdot a^{4}$ for $a=\sqrt[3]{\frac{1}{4}}$ with equality if and only if $G$ only has vertices of degree 1 and 4;

3) $S E I_{a}(G) \geq n \cdot \frac{4 a^{4}+2 a}{3}$ for $a \in\left(\sqrt[3]{\frac{1}{4}}, 1\right)$ with equality if and only if $G$ is a tree in which all vertices have degrees 1 and 4 .

Proof: In order to prove 1) and 2), it is sufficient to note that

1') $4 a^{4}<\min \left\{3 a^{3}, 2 a^{2}, a\right\}$ for $a \in\left(0, \sqrt[3]{\frac{1}{4}}\right)$

2) $4 a^{4}=a>\max \left\{2 a^{2}, 3 a^{3}\right\}$ for $a=\sqrt[3]{\frac{1}{4}}$.

Let us prove 3$)$. Suppose that $a \in\left(\sqrt[3]{\frac{1}{4}}, 1\right)$. If $G$ is a tree, then claim follows from Theorem 18. Hence, it is sufficient to prove that

$$
S E I_{a}(G)>n \cdot \frac{4 a^{4}+2 a}{3}
$$

for all cyclic chemical graphs with $n$ vertices. Suppose to the contrary, and let $G$ be a graph such that

$$
S E I_{a}(G) \leq n(G) \cdot \frac{4 a^{4}+2 a}{3}
$$

with the smallest value of $c=m-(n-1)$. Since $G$ is cyclic, $c>0$. Let $u v$ be the edge contained in some cycle and let $G^{+}$be a graph obtained from $G$ by eliminating the edge $u v$ and by adding one pendant vertex to each of vertices $u$ and $v$. Note that:

$$
S E I_{a}\left(G^{+}\right)=S E I_{a}(G)+2 \cdot a .
$$

Since $c\left(G^{+}\right)<c(G)$, it follows that

$$
\operatorname{SEI}_{a}\left(G^{+}\right)>(n(G)+2) \cdot \frac{4 a^{4}+2 a}{3} .
$$

On the other hand,

$$
\begin{aligned}
& \operatorname{SEI}_{a}\left(G^{+}\right)=S E I_{a}(G)+2 \cdot a \leq n(G) \cdot \frac{4 a^{4}+2 a}{3}+2 a< \\
& <(n(G)+2) \cdot \frac{4 a^{4}+2 a}{3} .
\end{aligned}
$$

This is a contradiction.

\section{CONCLUSIONS}

In this paper we have analyzed extremal properties of the variable sum exdeg index $S E I_{a}(G)$. We have found the graphs with the extremal graphs in the following classes of graphs (with given number of vertices):

1) class of all connected graphs

2) class of all trees

3) class of all unycyclic graphs

4) class of all chemical graphs

5) class of all chemical trees

6) class of all chemical unycyclic graphs

7) class of all graphs with given maximal degree

8) class of all graphs with given minimal degree

9) class of all trees with given number of pendant vertices

10) class of all connected graphs with given number of pendant vertices

for all $S E I_{a}$ indices such that $a>1$. In the case of $a \in(0,1)$, we have restricted our attention to chemical graphs, chemical trees and chemical unycyclic graphs. We leave the solution of the analogous problem in the remaining seven classes as an open problem.

Further, we propose solving the analogous set of problems for the two descriptors that have shown good predicitve properties in paper Ref. 3. Namely, we propose the study of the following variable descriptors:

- variable inverse sum deg index: $\sum_{u v \in E(G)} \frac{1}{d_{u}^{a}+d_{v}^{a}}$, $a \in \mathbb{R} \backslash\{0\} ;$

- variable inverse sum lodeg index:

$$
\sum_{u v E(G)} \frac{1}{\ln ^{a}\left(d_{u}\right)+\ln ^{a}\left(d_{v}\right)}, a \in \mathbb{R}^{+} .
$$

Further, we propose the study of the generalizations of discrete Adriatic indices that have shown good predictive properties in paper Ref. 1 . The problem regarding these generalizations are extensions of the open problems presented in paper Ref. 1. Namely, we propose the study of the following variable descriptors:

- variable Randić type lodeg index:

$$
\sum_{u v \in E(G)} \ln ^{a}\left(d_{u}\right) \cdot \ln ^{a}\left(d_{v}\right), a \in \mathbb{R}^{+}
$$


- variable Randić type di index: $\sum_{u v \in E(G)}\left(D_{x}^{a} D_{y}^{a}\right)$, $a \in \mathbb{R} \backslash\{0\} ;$

- Randić type exdi index: $\sum_{u v \in E(G)} a^{D_{u}} \cdot a^{D_{v}}$, $a \in \mathbb{R}^{+} \backslash\{1\} ;$

- variable sum lodeg index: $\sum_{u v \in E(G)}\left(\ln ^{a} d_{u}+\ln ^{a} d_{v}\right)=\sum_{v \in V(G)} d_{u} \ln ^{a} d_{u}$, $a \in \mathbb{R}^{+}$;

- variable inverse sum lodeg index: $\sum_{u v \in E(G)} \frac{1}{\ln ^{a}\left(d_{u}\right)+\ln ^{a}\left(d_{v}\right)}, \quad a \in \mathbb{R}^{+} ;$

- variable inverse sum deg: $\sum_{u v \in(G)} \frac{1}{d_{u}^{a}+d_{v}^{a}}$, $a \in \mathbb{R} \backslash\{0\} ;$

- variable misbalance lodeg index: $\sum_{u v \in E(G)}\left|\ln ^{a} d_{u}-\ln ^{a} d_{v}\right|, a \in \mathbb{R}^{+}$;

- $\quad$ variable misbalance deg index: $\sum_{u v \in E(G)}\left|d_{u}^{a}-d_{v}^{a}\right|$, $a \in \mathbb{R} \backslash\{0\} ;$

- variable misbalance exdeg index: $\sum_{u v \in E(G)}\left|a^{d_{u}}-a^{d_{v}}\right|, \quad a \in \mathbb{R}^{+} \backslash\{1\} ;$

- variable misbalance di index: $\sum_{u v \in E(G)}\left|D_{u}^{a}-D_{v}^{a}\right|$, $a \in \mathbb{R} \backslash\{0\} ;$

- variable min-max deg index: $\sum_{u v \in E(G)} \frac{\min \left\{d_{u}^{a}, d_{v}^{a}\right\}}{\max \left\{d_{u}^{a}, d_{v}^{a}\right\}}, a \in \mathbb{R}^{+}$

- variable min-max di index:

$$
\sum_{u v \in E(G)} \frac{\min \left\{D_{u}^{a}, D_{v}^{a}\right\}}{\max \left\{D_{u}^{a}, D_{v}^{a}\right\}}, a \in \mathbb{R}^{+} ;
$$

- variable max-min deg index:

$$
\sum_{u v \in E(G)} \frac{\max \left\{d_{u}^{a}, d_{v}^{a}\right\}}{\min \left\{d_{u}^{a}, d_{v}^{a}\right\}}, a \in \mathbb{R}^{+} ;
$$

- variable symmetric division deg index:

$$
\sum_{u v \in E(G)}\left(\frac{\min \left\{d_{u}^{a}, d_{v}^{a}\right\}}{\max \left\{d_{u}^{a}, d_{v}^{a}\right\}}+\frac{\max \left\{d_{u}^{a}, d_{v}^{a}\right\}}{\min \left\{d_{u}^{a}, d_{v}^{a}\right\}}\right), a \in \mathbb{R}^{+} .
$$

Acknowledgements. The partial support of Croatian Ministry of Science, Education and Sport (grants no. 177-00000000884 and 037-0000000-2779) is gratefully acknowledged.

Sarah Michele Rajtmajer's help considering English language is also gratefully acknowledged.

\section{REFERENCES}

1. D. Vukičević and M. Gašperov, Bond Additive Modeling 1 . Adriatic Indices, Croat. Chem. Acta 83 (3) (2010) 243-260.

2. D. Vukičević, Bond Additive Modeling 2. Mathematical properties of Max-min rodeg index, Croat. Chem. Acta 83 (3) (2010) 261-273.

3. D. Vukičević, Bond Aditive Modeling 3. QSPR and QSAR studies of variable Adriatic indices, Croat. Chem. Acta 83 (3) (2010) 349-351.

4. I. Gutman, B. Ruščić, N. Trinajstić, and C. F. J. Wilcox Jr, Chem. Phys. 62 (1975) 3399-3405.

5. S. Nikolić, G. Kovačević, A. Miličević, and N. Trinajstić, Croat. Chem. Acta 76 (2003) 113-124.

6. R. Todeschini and V. Consonni, Handbook of Molecular Descriptors, Wiley-VCH, Weinheim, 2000.

7. N. Trinajstić, Chemical Graph Theory, CRC Press, Boca Raton, 1992.

8. P. Hansen and D. Vukičević, Croat. Chem. Acta 80 (2007) $165-168$.

9. D. Vukičević and A. Graovac, MATCH Commun. Math. Comput. Chem. 57 (2007) 587-590.

10. D. Vukičević, MATCH Commun. Math. Comput. Chem. 57 (2007) 633-641.

11. D. Vukičević and A. Graovac, MATCH Commun. Math. Comput. Chem. 60 (2008) 37-44.

12. H. Hua, MATCH Commun. Math. Comput. Chem. 60 (2008) $45-56$. 